\title{
GPR Detection of Buried Symmetrically Shaped Mine-like Objects using Selective Independent Component Analysis
}

\author{
Brian Karlsen $^{a}$, Helge B.D. Sørensen ${ }^{a}$, Jan Larsen ${ }^{b}$, and Kaj B. Jakobsen ${ }^{a}$ \\ ${ }^{a}$ Ørsted•DTU, Technical University of Denmark \\ Ørsteds Plads, Building 348, DK-2800 Kongens Lyngby, Denmark \\ ${ }^{b}$ Informatics and Mathematical Modelling, Technical University of Denmark \\ Richard Petersens Plads, Building 321, DK-2800 Kongens Lyngby, Denmark
}

\begin{abstract}
This paper addresses the detection of mine-like objects in stepped-frequency ground penetrating radar (SFGPR) data as a function of object size, object content, and burial depth. The detection approach is based on a Selective Independent Component Analysis (SICA). SICA provides an automatic ranking of components, which enables the suppression of clutter, hence extraction of components carrying mine information. The goal of the investigation is to evaluate various time and frequency domain ICA approaches based on SICA. The performance comparison is based on a series of mine-like objects ranging from small-scale anti-personal (AP) mines to largescale anti-tank (AT) mines. Large-scale SF-GPR measurements on this series of mine-like objects buried in soil were performed. The SF-GPR data was acquired using a wideband monostatic bow-tie antenna operating in the frequency range $750 \mathrm{MHz}-3.0 \mathrm{GHz}$. The detection and clutter reduction approaches based on SICA are successfully evaluated on this SF-GPR dataset.
\end{abstract}

Keywords: time and frequency based ICA, Selective ICA, stepped-frequency, features, wideband, bow-tie antenna

\section{INTRODUCTION}

In recent years the development of signal processing techniques for automatic detection of anti-personal (AP) landmines from sensor signals has received significant interest. This paper focuses on an unambiguous detection of non-metallic AP-landmines in ground penetrating (GPR) radar signals using independent component analysis (ICA). The detection of non-metallic AP-landmines using a GPR is a non-trivial task due to weak scattering ${ }^{2,3}$. Nevertheless, the GPR is widely used as one of the main sensors in state-of-the-art AP-landmine detection systems $^{1}$. Most AP-landmines are buried close to the surface of the ground, and automatic object detection is hampered by the strong clutter from the ground surface. In general, the clutter that effects GPR can be defined as signals that are unrelated to the target scattering characteristics but occupy the same frequency band as the targets. Clutter can be caused by multiple reflections, e.g., in the antenna, between the antenna and the ground surface, and the non-landmine targets buried in the ground. However, on the detection of shallow buried non-metallic AP-landmines the ground surface clutter is the strongest and most significant clutter. Hence, to increase the detection of shallow buried objects, like the non-metallic AP-landmines, it is necessary to deploy proper clutter reduction and detection methods.

The literature suggests a number of clutter reduction and detection methods, such as likelihood ratio testing ${ }^{4}$, parametric system identification ${ }^{5-8}$, wavelet packet decomposition ${ }^{9,10}$, subspace techniques ${ }^{11-15}$, and simple mean subtraction ${ }^{2}$. However, many of these fail to detect shallow buried AP-landmines, mostly because of the statistical nature of the clutter, e.g., the ground surface is not perfectly flat nor even relative smooth. Another problem is that many of the methods use reference-signal estimates or templates of the signature of the target or the clutter. These reference-signals and templates are used to remove the signal that are unrelated to the target signal signatures. However, a target signal which has little correlation with the reference-signals or templates

Further author information on: $B K$ : brk@oersted.dtu.dk, www.oersted.dtu.dk; $H B D S$ : hbs@oersted.dtu.dk, www.oersted.dtu.dk; JL: jl@imm.dtu.dk, www.imm.dtu.dk/ jl; KBJ: kbj@oersted.dtu.dk, www.oersted.dtu.dk 
may not be detected, hence, be classified as clutter. Further, the approaches are also often sensitive to unknown disturbances in the soil and in the target and clutter signal signatures. This is in particular a problem in the detection of weak scatters, like the non-metallic AP-landmine.

To overcome this problem methods based on blind source signal processing techniques are used. In many signal processing applications the sensor cannot directly measure the signals of interest. However, in general we have access to a linear mixture of the signals, where the mixing coefficients are unknown. In this kind of a signal processing problem, we would like to recover the original signals (or sources) in a blind manner without knowing the mixing coefficients. This method is known as blind source separation (BSS). Here, the sources are related to the underlying mine scatter signals we want to recover. Recently, we have suggested promising BSS methods ${ }^{12,13}$ for clutter reduction and non-metallic AP-landmine detection based on decomposition of the GPR signals into clutter and landmine signals using principal component analysis (PCA) and independent component analysis (ICA). Both methods are unsupervised methods, i.e., we do not use explicit knowledge of object type, but use the fact that target and the clutter signals possess different statistical nature and are independent.

In this paper we extent recent work by considering both time-domain and frequency-domain GPR based ICA approaches. Further, we suggest a component selection technique for enhanced identification of non-metallic AP-landmines. In Section 2 we give a review on the proposed ICA approach for GPR signals. In section 3 we extent the ICA approach with different mixture models for GPR signals. A scheme for selection of relevant ICA components of non-metallic AP-landmines is presented in section 4. Finally, section 5 provides a comparative study of the presented methods, which are tested on GPR signals collected at an indoor GPR measurement facility at the Technical University of Denmark.

\section{INDEPENDENT COMPONENT ANALYSIS OF GPR SIGNALS}

The most general BSS problem can be formulated as follows. We observe the output of a linear or nonlinear system, where its inputs are generated from a number of source signals. An example of such a system could be a GPR-system, where the source signals are the target and clutter signals, and the received GPR signals are the output of the system which is a mixture of the source signals given by the target and clutter signals. In the application of clutter reduction and target detection, one would like to separate the source signals into target signal sources and clutter signal sources, and then use the target signal sources for detection. Using BSS, the separation can be done in a blind manner if we assume that the source signals are mutually independent. The independence assumption is referred to as ICA. Hence, in the application of landmine detection using GPR we decompose the received GPR signals, into independent components, i.e., independent source signals. Some of these components will mainly include clutter signals and others mainly landmine signals. By removal of the independent components that forms the clutter signal space, it is possible to enhance the detection of the landmines.

There exist many different mathematical or physical models in the mixing process of unknown independent source signals. Therefore, a given mixture model depends on the specific application. In this paper we focus on the blind separation of noise-free linear instantaneous mixtures of independent source signals. This mixture model has shown good results in the application of landmine detection using GPR. The linear instantaneous mixture model can be expressed by

$$
\boldsymbol{X}=\boldsymbol{A} \boldsymbol{S}=\sum_{i=1}^{K} \boldsymbol{a}_{i} \boldsymbol{s}_{i}^{\top} \quad \text { and } \quad X_{p, n}=\sum_{i=1}^{K} A_{p, i} S_{i, n}
$$

where $\boldsymbol{X}=\left\{X_{p, n}\right\}=\left[\boldsymbol{x}_{1}, \boldsymbol{x}_{2}, \cdots, \boldsymbol{x}_{N}\right]$ is a $P \times N$ dimensional signal matrix that spans the space of the received GPR signals, $\boldsymbol{A}=\left\{A_{m, i}\right\}=\left[\boldsymbol{a}_{1}, \boldsymbol{a}_{2}, \cdots, \boldsymbol{a}_{K}\right]$ is a general mixing matrix of dimension $P \times K$ and $\boldsymbol{S}=\left\{S_{i, n}\right\}=$ $\left[\boldsymbol{s}_{1}, \boldsymbol{s}_{2}, \cdots, \boldsymbol{s}_{K}\right]^{\top}$ is a set of $K$ independent source signals. $\boldsymbol{S}$ has the dimension $K \times N$. $P$ is the number of sensors, $N$ the number of samples in each sensor signal, and $K$ the number of independent sources. That is, from the linear mixture model expressed by eq. (1) the ICA forms a subspace of independent source signals, $\boldsymbol{S}$. In other words, ICA decomposes $\boldsymbol{X}$ into $K$ independent source signals, $\boldsymbol{S}$. 
In order to recover the original independent source signals, $\boldsymbol{S}$, from the observed mixture, $\boldsymbol{X}$, we use a simple linear separating system expressed by

$$
\widehat{\boldsymbol{S}}=\boldsymbol{W} \boldsymbol{X}
$$

where $\widehat{\boldsymbol{S}}=\left\{S_{i, n}\right\}=\left[\widehat{\boldsymbol{s}}_{1}, \widehat{\boldsymbol{s}}_{2}, \cdots, \widehat{\boldsymbol{s}}_{K}\right]^{\top}$ is the $K \times N$ dimensional matrix of $K$ estimated source signals, and $\boldsymbol{W}$ is an estimated unmixing matrix, which is the pseudo inverse of $\boldsymbol{A}, \boldsymbol{W}^{\sharp}=\boldsymbol{A}$. That is, from eq. (2) we are able to estimate $\boldsymbol{A}$ and $\boldsymbol{S}$ up to scaling factors and permutations of the source signals. In this paper we do not focus on algorithms for estimating $\boldsymbol{A}$ and $\boldsymbol{S}$. However, the literature provides a number of algorithms ${ }^{16,17}$. Some deploy higher (or lower) order moments of non-Gaussian sources, whereas others use the correlation of the source signals. We deploy a member from each family: the widely used Bell-Sejnowski (BS-ICA) algorithm using natural gradient learning $^{18}$, and the Molgedey-Schuster ${ }^{19,20}$ (MS-ICA) algorithm using the implementations provided in the DTU-Toolbox ${ }^{21}$.

Pre-whitening of the GPR signals is optional in order to improve convergence speed for ill-conditioned problems. We provide PCA which is a orthogonal transform and decorrelation approach for pre-whitening of the GPR signals. This method can also be used in BSS problems for source separation. Here, the major difference from ICA is that the sources from PCA, the so called principal components, are constrained to be uncorrelated rather than mutually independent. As input to the ICA we first employ the PCA on the GPR signals. PCA can be executed using singular value decomposition (SVD) expressed by

$$
\boldsymbol{X}=\boldsymbol{U} \boldsymbol{D} \boldsymbol{V}^{\top}=\sum_{i=1}^{N} \boldsymbol{u}_{i} D_{i, i} \boldsymbol{v}_{i}^{\top} \quad \text { and } \quad X_{p, n}=\sum_{i=1}^{N} U_{p, i} D_{i, i} V_{n, i}
$$

where the $P \times N$ matrix $\boldsymbol{U}=\left\{U_{p, i}\right\}=\left[\boldsymbol{u}_{1}, \boldsymbol{u}_{2}, \cdots, \boldsymbol{u}_{N}\right]$ and the $N \times N$ matrix $\boldsymbol{V}=\left\{V_{n, i}\right\}=\left[\boldsymbol{v}_{1}, \boldsymbol{v}_{2}, \cdots, \boldsymbol{v}_{N}\right]$ represent orthonormal basis vectors, i.e., eigenvectors of the symmetric matrices $\boldsymbol{X} \boldsymbol{X}^{\mathrm{T}}$ and $\boldsymbol{X}^{\mathrm{T}} \boldsymbol{X}$, respectively. $\boldsymbol{D}=D_{i, i}$ is an $N \times N$ diagonal matrix of singular values ranked in decreasing order, as shown by $D_{i, i} \geq$ $D_{i+1, i+1}, \forall i \in[1 ; N-1]$. The SVD identifies a set of uncorrelated time signals, the principal components (PC's): $\boldsymbol{y}_{i}=D_{i, i} \boldsymbol{v}_{i}$, enumerated by the component index $i=1,2, \ldots, N$ and $\boldsymbol{y}_{i}=\left[y_{i}(1), \cdots, y_{i}(N)\right]^{\top}$. The dimension of the PCA data set will be $K \leq N$. That is, we model $\boldsymbol{X}$ only from non-zero eigenvalues ${ }^{22}$ and further have the possibility of projecting onto a subspace. Pre-whitening and subspace projection of $\boldsymbol{X}$ is obtained by

$$
\widetilde{\boldsymbol{X}}=\widetilde{\boldsymbol{U}}^{\top} \boldsymbol{X}
$$

where $\widetilde{\boldsymbol{U}}=\left[\boldsymbol{u}_{1}, \boldsymbol{u}_{2}, \cdots, \boldsymbol{u}_{K}\right]$ is $P \times K$ and $\widetilde{\boldsymbol{X}}$ is a $K \times N$ matrix. Hence, after pre-whitening and projection the $K \times K$ ICA problem with mixing matrix $\boldsymbol{\Phi}$ is

$$
\widetilde{\boldsymbol{X}}=\boldsymbol{\Phi} \boldsymbol{S} \quad, \quad \widehat{\boldsymbol{S}}=\boldsymbol{W} \boldsymbol{X}=\boldsymbol{\Phi}^{-1} \widetilde{\boldsymbol{U}}^{\top} \boldsymbol{X}
$$

In the application of landmine detection in GPR signals, the ICA can be used to detect landmines and reduce clutter. By selecting (see further section 4) components which mainly carry mine information, say $\widehat{\boldsymbol{s}}_{k}$, we can remove clutter. The reconstructed signal space in the original GPR signal space is then

$$
\widehat{\boldsymbol{x}}_{k}=\tilde{\boldsymbol{U}} \boldsymbol{\Phi} \widehat{\boldsymbol{s}}_{k}
$$

\section{THE GPR SIGNAL MATRIX}

The signal matrix, $\boldsymbol{X}$, that spans the signal space of the received GPR signals can be constructed in different ways. Hence four different mixture models based on eqs. (1)-(5).

\subsection{Time-Time: Time independence of GPR time signals}

The mixture model for time independence of GPR time signals embodies the assumption that the GPR time signals is a linear mixture of $K$ independent GPR time signal sequences. Hence, we have the linear mixture model expressed by $\boldsymbol{X}_{1}=\boldsymbol{A}_{1} \boldsymbol{S}_{1}=\widetilde{\boldsymbol{U}}_{1} \boldsymbol{\Phi}_{1} \boldsymbol{S}_{1}$, where $\boldsymbol{A}_{1}$ is a $P \times K$ dimensional mixture matrix, $\boldsymbol{S}_{1}$ is a $K \times N$ dimensional set of $K$ independent GPR time signal sequences, and $\boldsymbol{X}_{1}$ is a $P \times N$ dimensional signal matrix 
that spans the received GPR time signal $\boldsymbol{X}_{1}=\left\{X_{1, p, n}\right\}$, where $P$ is the number of sensor signals. The sensor signals are given by the GPR time signals, which are received by scanning the GPR above the ground surface in the $\mathrm{x}$ - and $\mathrm{y}$-direction. $N$ is the number of samples in each of the received GPR time signals. Hence, $x_{1, p}(n)$ is sample $n$ of the GPR time signal received at the antenna located at position $(\mathrm{x}, \mathrm{y})=((i-1) \Delta \mathrm{x},(j-1) \Delta \mathrm{y})$, where $i=1,2, \cdots, I$, and $j=1,2, \cdots, J . \Delta \mathrm{x}$ and $\triangle \mathrm{y}$ are the antenna location step size in the $\mathrm{x}$ - and $\mathrm{y}$-direction, respectively, and $p=i+(j-1) I . I$ and $J$ are the numbers of antenna locations in the $\mathbf{x}$ - and $\mathbf{y}$-direction, respectively.

\subsection{Time-Spatial: Space independence of GPR time signals}

The mixture model for space independence of GPR time signals embodies the assumption that the GPR time signals is a linear mixture of $K$ independent xy-images. Hence, we have the linear mixture model expressed by $\boldsymbol{X}_{2}=\boldsymbol{A}_{2} \boldsymbol{S}_{2}=\widetilde{\boldsymbol{U}}_{2} \boldsymbol{\Phi}_{2} \boldsymbol{S}_{2}$, where $\boldsymbol{A}_{2}$ is a $K \times K$ dimensional mixture matrix, $\boldsymbol{S}_{2}$ is a $K \times P$ dimensional set of $K$ independent GPR $x y$-images and $\boldsymbol{X}_{2}$ is a $N \times P$ dimensional signal matrix that spans the GPR time signal space observed where $P$ is the number of sensor signals and $N$ is the number of time samples. That is, $\boldsymbol{X}_{2}=\boldsymbol{X}_{1}^{\top}$. The next two models we are considering frequency models of the GPR signals. Since stepped-frequency GPR measurement is a complex frequency representation we could also consider the ICA a decomposing the received spectrum into characteristic mine and clutter spectra. For simplicity we will consider only the magnitude spectrum hence using real ICA models.

\subsection{Frequency-Frequency: Frequency independence of GPR frequency signals}

The mixture model for frequency independence of the GPR frequency spectra embodies the assumption that the GPR frequency spectra is a linear mixture of $K$ independent frequency spectra. Hence, we have the linear mixture model expressed by $\boldsymbol{X}_{3}=\boldsymbol{A}_{3} \boldsymbol{S}_{3}=\widetilde{\boldsymbol{U}}_{3} \boldsymbol{\Phi}_{3} \boldsymbol{S}_{3}$, where $\boldsymbol{A}_{3}$ is a $K \times K$ dimensional mixture matrix, $\boldsymbol{S}_{3}$ is a $K \times N$ dimensional set of $K$ independent GPR magnitude frequency spectra and $\boldsymbol{X}_{3}$ is a $P \times N$ dimensional signal matrix that spans the GPR frequency spectra observed. For this mixture model $\boldsymbol{X}_{3}$ is constructed as $\boldsymbol{X}_{3}=\left\{X_{3, p, n}\right\}$ where $P$ is the number of sensor signals, which are received by scanning the GPR above the ground surface in the $\mathrm{x}$ - and $\mathrm{y}$-direction. $N$ is the number of frequencies in each of the received frequency spectra, and $x_{1, i}(n)$ is the amplitude of the frequency bin $n$ in the GPR frequency spectrum received at the GPR antenna located at position $(\mathrm{x}, \mathrm{y})=((i-1) \triangle \mathrm{x},(j-1) \triangle \mathrm{y})$, where $i=1,2, \cdots, I$, and $j=1,2, \cdots, J . \Delta \mathrm{x}$ and $\triangle \mathrm{y}$ are the antenna location step size in the $\mathrm{x}$ - and $\mathrm{y}$-direction, respectively, and $p=i+(j-1) I . I$ and $J$ is the number of antenna locations in the $\mathrm{x}$ - and $\mathrm{y}$-direction, respectively.

\subsection{Frequency-Spatial: Space independence of GPR time frequency signals}

The mixture model for space independence of GPR frequency signals embodies the assumption that the GPR frequency signals is a linear mixture of $K$ independent magnitude GPR frequency xy-images. This model is the transpose of mixture model 3, thus $\boldsymbol{X}_{4}=\boldsymbol{X}_{3}^{\top}$. The linear mixture model is expressed by $\boldsymbol{X}_{4}=\boldsymbol{A}_{4} \boldsymbol{S}_{4}=\widetilde{\boldsymbol{U}}_{4} \boldsymbol{\Phi}_{4} \boldsymbol{S}_{4}$, where $\boldsymbol{A}_{4}$ is a $K \times K$ dimensional mixture matrix, $\boldsymbol{S}_{4}$ is a $K \times P$ dimensional set of $K$ independent GPR magnitude frequency xy-images and $\boldsymbol{X}_{4}$ is a $N \times P$ dimensional signal matrix that spans the observed GPR magnitude frequency signal space, where $P$ is the number of sensor signals and $N$ is the number of frequency bins.

\section{SCHEME FOR SELECTIVE INDEPENDENT COMPONENT ANALYSIS}

The ranking and selection of relevant components can be done in many different ways. We suggest a simple method based on a measure of the fourth-order statistics of the extracted independent sources ${ }^{23}$. Similar to the PCA where the orthogonal sources are sorted by the variance (second-order statistics) we can sort the independent sources according to non-Gaussianity. It turns out that independent components with a high contrast and with high non-Gaussian structure carry mine information whereas clutter components are more Gaussian. A way to estimate non-Gaussianity is by evaluating the normalized kurtosis,

$$
\kappa_{4}\left(\widehat{s}_{i}\right)=\frac{E\left\{\left|\widehat{s}_{i}\right|^{4}\right\}}{E^{2}\left\{\left|\widehat{s}_{i}\right|^{2}\right\}}-3 .
$$

Sorting the independent according to normalized kurtosis and selecting components with $\kappa_{4}>\delta$, where $\delta>0$ is a given threshold, provides the selection of mine components. 


\section{EXPERIMENTS AND RESULTS}

In order to get an idea about the effectiveness of the proposed ICA mixture models and the scheme for the ranking of the IC's for clutter reduction and non-metallic AP-landmine detection, we have performed studies on field-test stepped-frequency GPR signals. The field-test data was collected using a monostatic bow-tie antenna operating in the frequency range $750 \mathrm{MHz}-3.0 \mathrm{GHz}$. The data was acquired using a HP8753A network analyzer. In a measurement area of $101 \mathrm{~cm} \times 101 \mathrm{~cm}$ non-metallic cylinders of different size and with different content of non-metallic material were buried in different depths. In table 1 are the technical specification of the the

\begin{tabular}{|l||l|l||l|l|}
\hline Target & Cyl. no. 1 & Cyl. no. 2 & Cyl. no. 3 & Cyl. no. 4 \\
\hline Diameter & $15 \mathrm{~cm}$ & $15 \mathrm{~cm}$ & $5 \mathrm{~cm}$ & $5 \mathrm{~cm}$ \\
\hline Height & $5 \mathrm{~cm}$ & $5 \mathrm{~cm}$ & $5 \mathrm{~cm}$ & $5 \mathrm{~cm}$ \\
\hline Content & Air & Beeswax & Air & Beeswax \\
\hline Container & Plastic & Plastic & Plastic & Plastic \\
\hline
\end{tabular}

Table 1. Test objects used for testing the proposed ICA mixture models.

buried non-metallic cylinders listed. Half of them were with no contents (air) and the other half were filled with beeswax. All the cylinders were buried in the center of the measurement area in relative dry soil $0 \mathrm{~cm}$, $5 \mathrm{~cm}$, and $10 \mathrm{~cm}$ below the surface. The relative permittivity of the soil was $\varepsilon_{r}=2.8$, and was estimated using a loop antenna ${ }^{24}$. The measurement area was scanned and SF-GPR signals were collected at every antenna positions located $(\triangle \mathrm{x}=1 \mathrm{~cm}) \times(\triangle \mathrm{y}=1 \mathrm{~cm})$ from each other. The number of frequencies were 601 . That is, $\triangle f=3.75 \mathrm{MHz}$. Before the ICA methods were deployed on the SF-GPR data, the SF-GPR data were noise reduced by smoothing the discrete spectrum of 601 frequencies measured at each GPR antenna location. For the Time-Time and Time-Space ICA mixture models the frequency-domain data were down-modulated to the base-band and Fourier-transformed to the time-domain using a sampling frequency of $30.72 \mathrm{GHz}$.

The four ICA mixture models were tested on the SF-GPR data using BS-ICA and MS-ICA. In figure 1 to figure 8 are selected results shown for BS-ICA and MS-ICA. The selected results shown are results from cylinder no. 1 and cylinder no. 2. To get the results we first removed the mean value from the SF-GPR signals and then deployed PCA as pre-whitening (see eq.4). After PCA the most noisy PCA subspaces were removed. From the PCA we ended up with 30-70 subspaces describing $100 \%$ of the SF-GPR data depending on the data example. We kept the first 20 PCA subspaces and used these subspaces as input for the BS-ICA and MS-ICA. After applying BS-ICA and MS-ICA we ranked the IC's after highest normalized kurtosis (see section 4). Within the IC's with highest kurtosis we did the selection of relevant IC's for reconstruction. In this way we have reduced the PCA subspace of up to 70 subspaces down to about 5 selective IC subspaces containing mainly landmine-like information.

As a performance measure on the reduction of clutter and detection, the area under the ROC curve is used. For all the considered examples the area under the ROC curve is listed in table 2. The closer to 1 the area under the ROC curve is the better the performance we have. From this it is clear that some of the ICA mixture models are better than others. In general, the ROC curve shows the performance of a particular detector or classifier. However, in this paper the ROC curves shown, and the area under the ROC curves, are more a measure on the clutter reduction, i.e., the signal-to-clutter radio. That is, in the results we know were the landmine is buried. Hence, by a threshold on the sum-variance image $\left(I_{i, j}=\operatorname{diag}\left(\widehat{\boldsymbol{x}}_{k}^{2}\right)\right)$ of each reconstructed signal matrices we can classify the image into clutter and landmine from the knowledge on were the landmines are buried. By changing the threshold the ROC curve is constructed. That is, the ROC curve is more a measure on how good the selected landmine signals are separated from the clutter, rather than a measure in general on classification or detection.

\subsection{Time-Time Results}

In the Time-Time ICA mixture model $\left(\boldsymbol{X}_{1}\right)$ we seek for time independence of time signals. In figure 1 and 2 are two examples of extracted IC's from the ICA mixture model shown. In figure 1 are results for BS-ICA shown, and in figure 2 are results for MS-ICA shown. For both examples the first 6 IC's (time signal sequences) and the corresponding mixture coefficients (eigenimages) are shown. From the results of the BS-ICA we have rather 
peaked IC's, and localized signatures in some of the eigenimages. In particular source no. 2 and no. 6 show landmine-like signatures in the eigenimages. Hence, they are used for reconstruction of the clutter reduced signal matrix. Further, the IC's peak at a depth that corresponds to the burial depth of the cylinder $(0 \mathrm{~cm}=2.5 \mathrm{~ns})$. The results from the MS-ICA are different from the BS-ICA. This is due to fact that MS-ICA is based on decorrelation of delayed time signals. Therefore, we get more correlated IC's which are independent from each other. Moreover, BS-ICA gives as non-Gaussian signals as possible. Hence, they are rather peaked. In general, the GPR time signals are peaked and not as correlated as the sources from the MS-ICA. Therefore, BS-ICA gives satisfactory results. This can also be seen from the results listed in table 2 . The results show that BS-ICA deployed on the ICA mixture model gives a satisfactory separation and detection on both cylinder no. 1 and no. 2 .

\subsection{Time-Spatial Results}

In the Time-Spatial ICA mixture model $\left(\boldsymbol{X}_{2}\right)$ we seek for spatial independence of time signals. In figure 1 and 2 are two examples of extracted IC's for the ICA mixture model shown. In figure 1 are results for BS-ICA shown and in figure 2 are results for MS-ICA shown. For both examples the first 6 IC's (eigenimages) and the corresponding mixture coefficients (time signal sequences) are shown. From the BS-ICA we get rather peaked IC's. Hence we have IC's with strong landmine signatures. In particular component no. 1 shows strong landminelike signatures. The corresponding mixer coefficients, which in this case are time signal signatures also show strong landmine-like signatures, i.e., they are peaked. The results from MS-ICA are non-satisfactory. Due to the fact that the buried cylinders are weak scatters it is hard for MS-ICA to enhance, i.e., through decorrelation, the spatial signatures without enhancing the clutter. Hence, no clutter reduction. From the results shown in table 2 it is clear that ICA mixture model gives non-satisfactory results for MS-ICA on weak scatters. However, on a strong scatter (cyl. no. 2) both BS-ICA and MS-ICA have a satisfactory performance.

\subsection{Frequency-Frequency Results}

In the Frequency-Frequency ICA mixture model $\left(\boldsymbol{X}_{3}\right)$ we seek for frequency independence of the frequency spectra. In figure 1 and 2 are two examples of extracted IC's for the ICA mixture model shown. In figure 1 are results for BS-ICA shown and in figure 2 are results for MS-ICA shown. For both examples the first 6 IC's (frequency spectra) and the corresponding mixture coefficients (eigenimages) are shown. From the BS-ICA we get rather peaked IC's. However, the physical signature of the frequency spectra is not peaked signals. Hence, the BS-ICA will extract IC's that may have non-physical signatures. In this example it is clear that we do not extract any landmine-like information. However, the MS-ICA is able to extract some landmine information. Because of the fact that the frequency spectra measured at each SF-GPR antenna position is rather correlated we get IC's that are similar to the real frequency spectra signature. Hence, good separation. From the results shown in table 2 it is clear that the ICA model gives a satisfactory performance on weak scatters for MS-ICA only. For strong scatters both MS-ICA and BS-ICA shows good results.

\subsection{Frequency-Spatial Results}

In Frequency-Spatial ICA mixture model $\left(\boldsymbol{X}_{4}\right)$ we seek for spatial independence of frequency spectra. In figure 1 and 2 are two examples of extracted sources for the ICA mixture model shown. In figure 1 are results for BS-ICA shown and in figure 2 are results for MS-ICA shown. For both examples the first 6 IC's (eigenimages) and the corresponding mixture coefficients (frequency spectra) are shown. From the BS-ICA we get rather peaked IC's. Hence, we have strong landmine signatures in the eigenimages. In particular component no. 1 shows strong landmine-like signatures. The frequency spectra shows a rather physical signature. The results from the MSICA are similar. From the results shown in table 2 it is clear that the ICA mixture model gives a satisfactory performance on weak and strong scatters for both MS-ICA and BS-ICA. 
\begin{tabular}{l|l} 
Frequency-Spatial, BS-ICA & Frequency-Frequency, BS-ICA
\end{tabular}

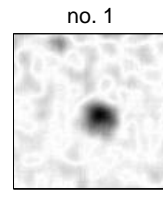

no. 4

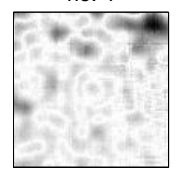

no. 1
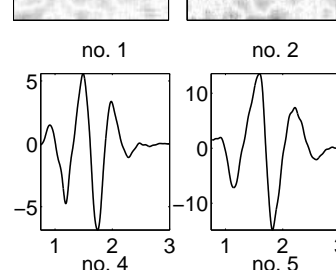

no. 2
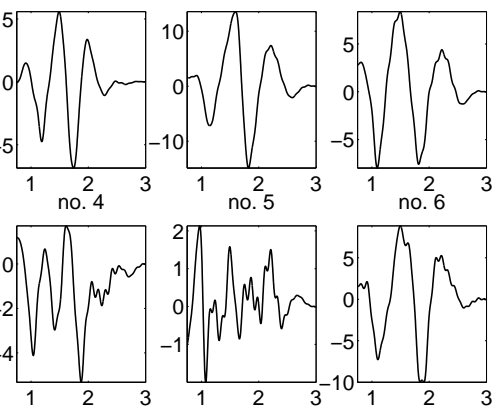

f $[\mathrm{GHz}]$

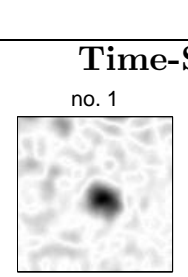

no. 4

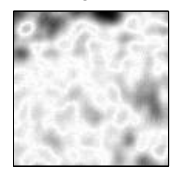

no. 1

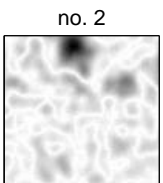

no. 5

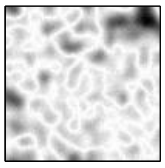

no. 2
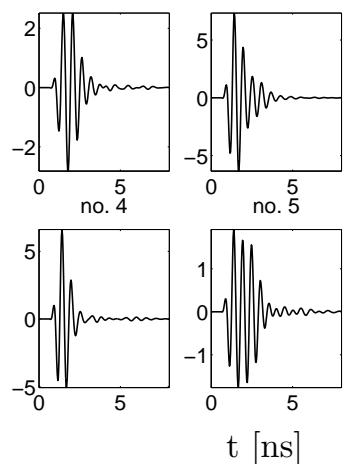

no. 6

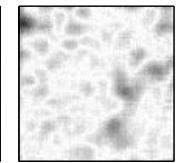

no. 3

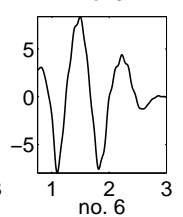

BS-ICA
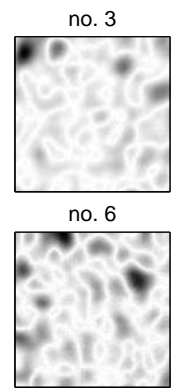

no. 3
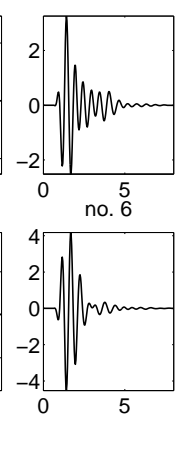

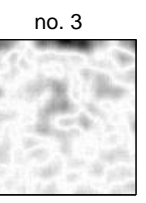

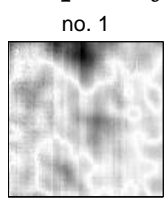

no. 4

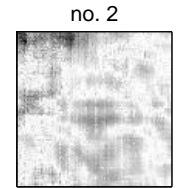

no. 5
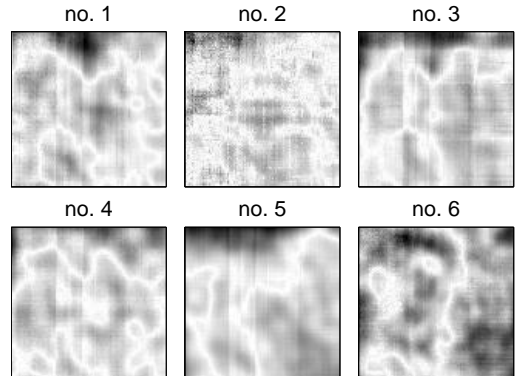

no. 6
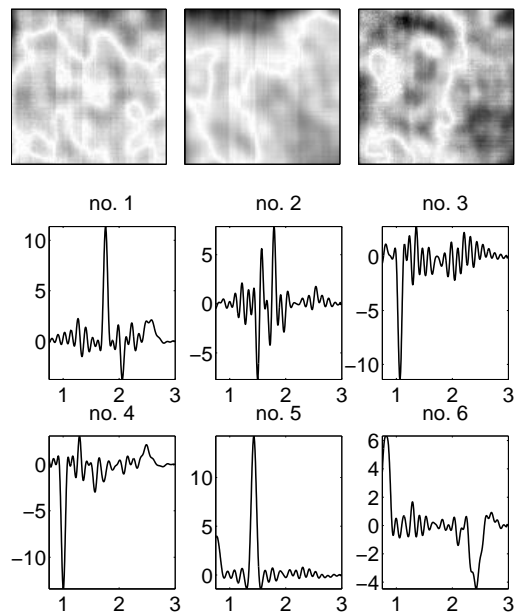

$f[\mathrm{GHz}]$
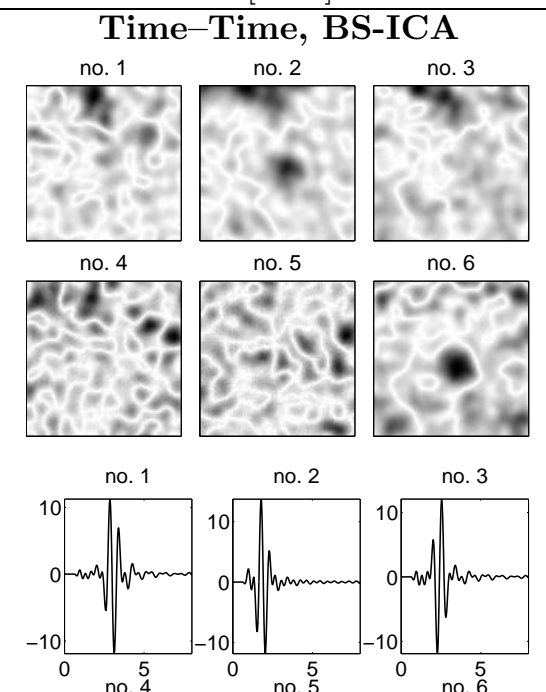

no. 2
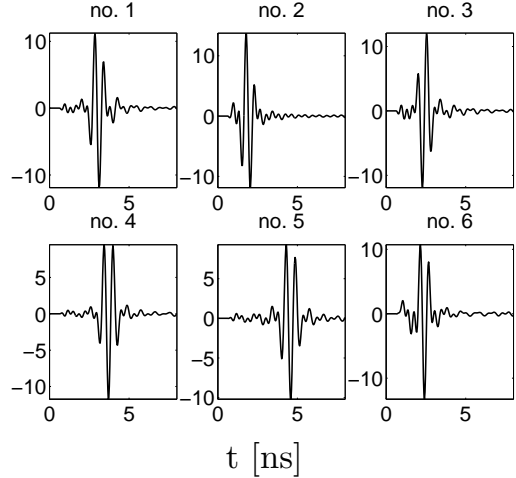

Figure 1. Results from BS-ICA deployed on the four ICA mixture models. The SF-GPR data used are measurements on cylinder no. 2 buried $0 \mathrm{~cm}$. Notice that for BS-ICA the Frequency-Frequency ICA mixture model have a non-satisfactory separation of the SF-GPR frequency signals. This is due to the fact that the frequencies in the frequency spectrum are not in general independent from each other. 
Frequency-Spatial, MS-ICA

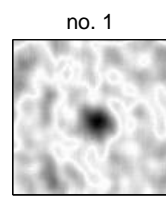

no. 4

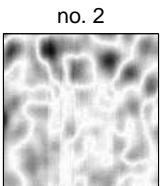

no. 5

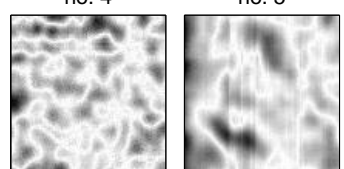

no. 1
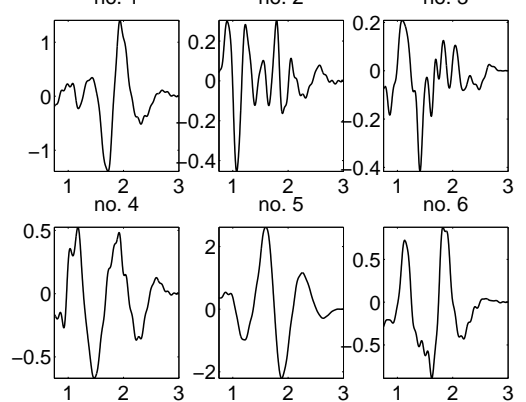

f $[\mathrm{GHz}]$

Time-Spatial, MS-ICA

no. 1

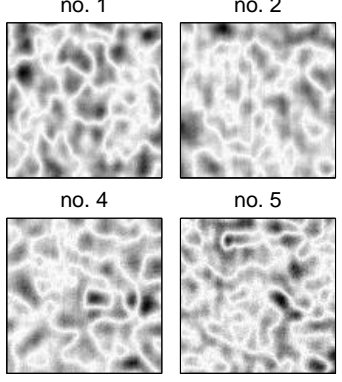

no. 1
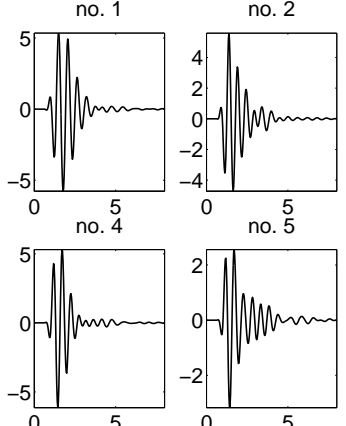

t [ns]
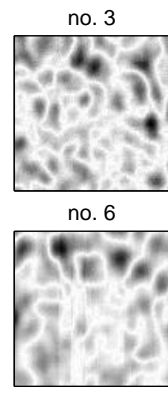

no. 3

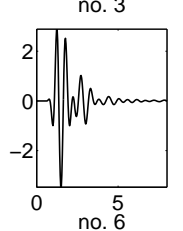

Frequency-Frequency, MS-ICA
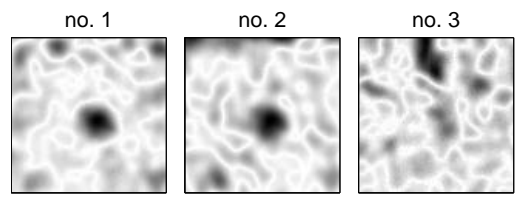

no. 5
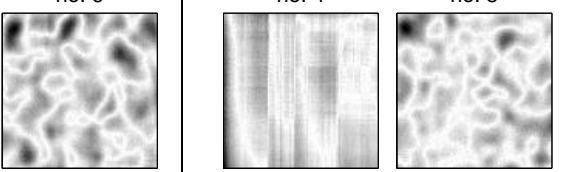

no. 6

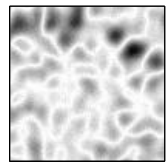

no. 1

no. 2

no. 3
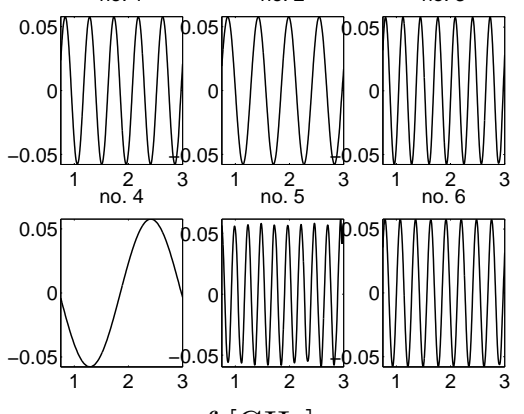

$\mathrm{f}[\mathrm{GHz}]$

Time-Time, MS-ICA

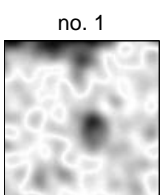

no. 2

no. 3

no. 4
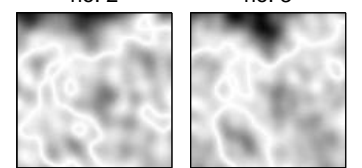

no. 5

no. 6
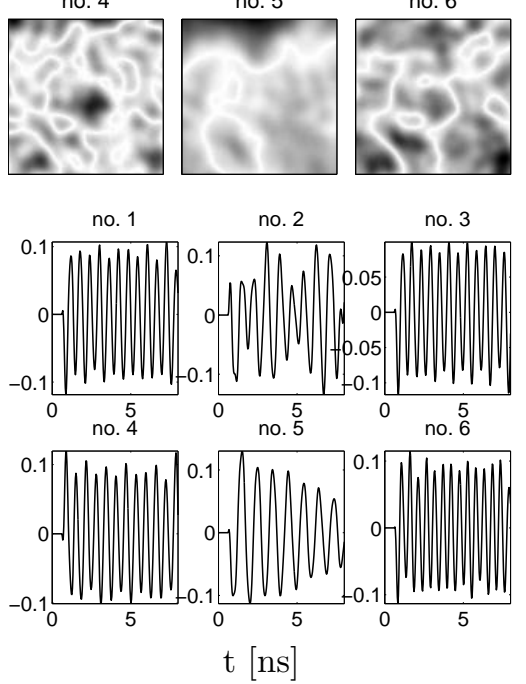

Figure 2. Results from MS-ICA deployed on the four ICA mixture models. The SF-GPR data used are measurements on the cylinder no. 2 buried $0 \mathrm{~cm}$ under the surface. Notice that the MS-ICA for the Time-Spatial ICA mixture model have a non-satisfactory performance due to weak scatters. 


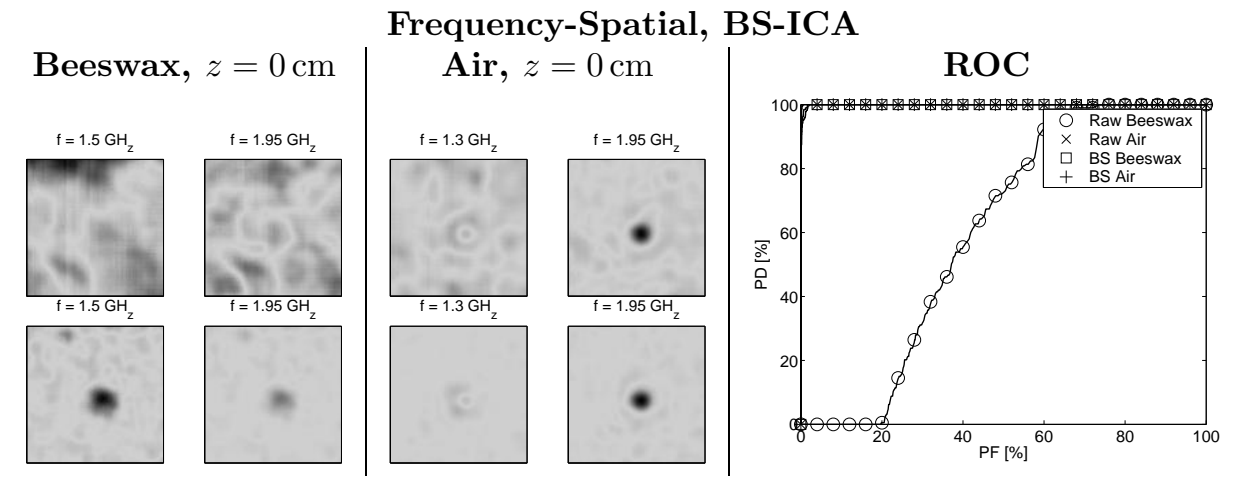

Figure 3. The first row shows the raw SF-GPR data at the frequencies $1.5 \mathrm{GHz}$ and $1.95 \mathrm{GHz}$ for cyl. no. 1 buried $0 \mathrm{~cm}$ and $1.3 \mathrm{GHz}$ and $1.95 \mathrm{GHz}$ for cyl. no. 2 buried $0 \mathrm{~cm}$. The second row shows the reconstructed data using BS-ICA on the Frequency-Spatial ICA mixture model. The images and the ROC curves show that the detection is improved.

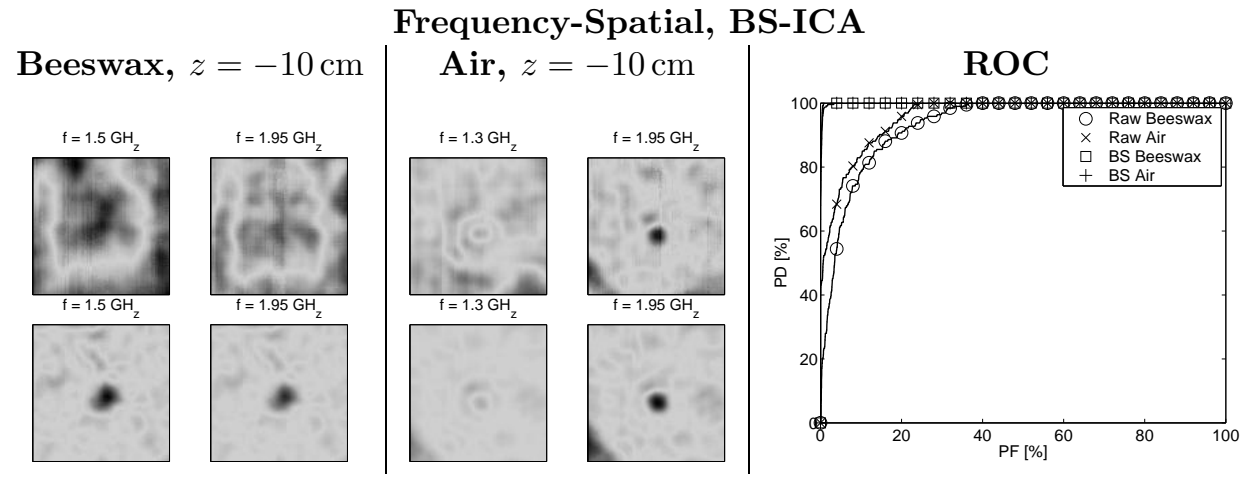

Figure 4. The first row shows the raw SF-GPR data at the frequencies $1.5 \mathrm{GHz}$ and $1.95 \mathrm{GHz}$ for cyl. no. 1 buried $10 \mathrm{~cm}$ and $1.3 \mathrm{GHz}$ and $1.95 \mathrm{GHz}$ for cyl. no. 2 buried $10 \mathrm{~cm}$. The second row shows the reconstructed data using BS-ICA on the frequency-Spatial ICA mixture model. The images and the ROC curves show that the detection is improved.

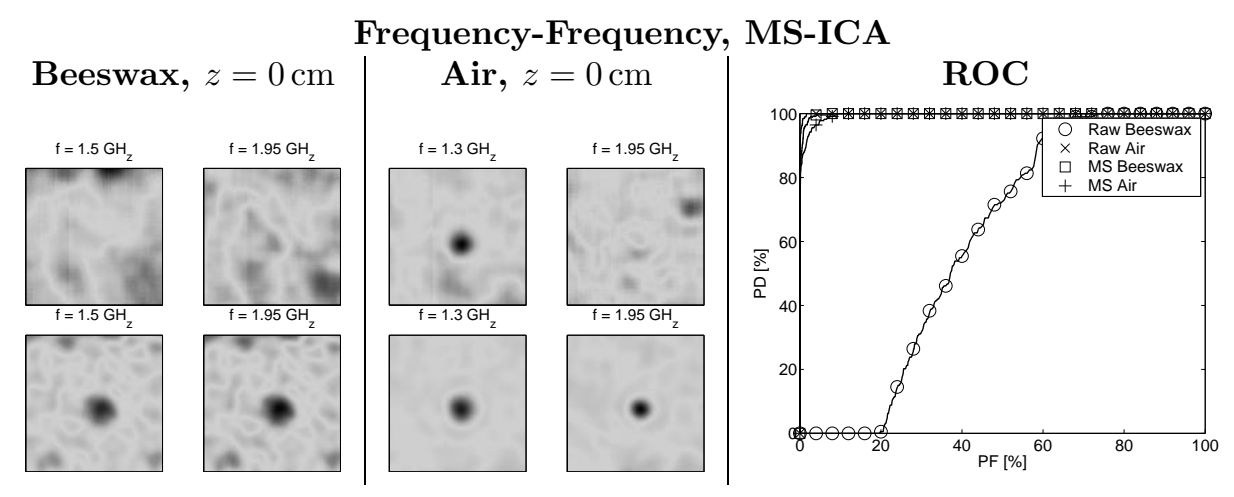

Figure 5. The first row shows the raw SF-GPR data at the frequencies $1.5 \mathrm{GHz}$ and $1.95 \mathrm{GHz}$ for cyl. no. $1 \mathrm{buried} 0 \mathrm{~cm}$ and $1.3 \mathrm{GHz}$ and $1.95 \mathrm{GHz}$ for cyl. no. 2 buried $0 \mathrm{~cm}$. The second row shows the reconstructed data using MS-ICA on the frequency-frequency ICA mixture model. The images and the ROC curves show that the detection is improved. 


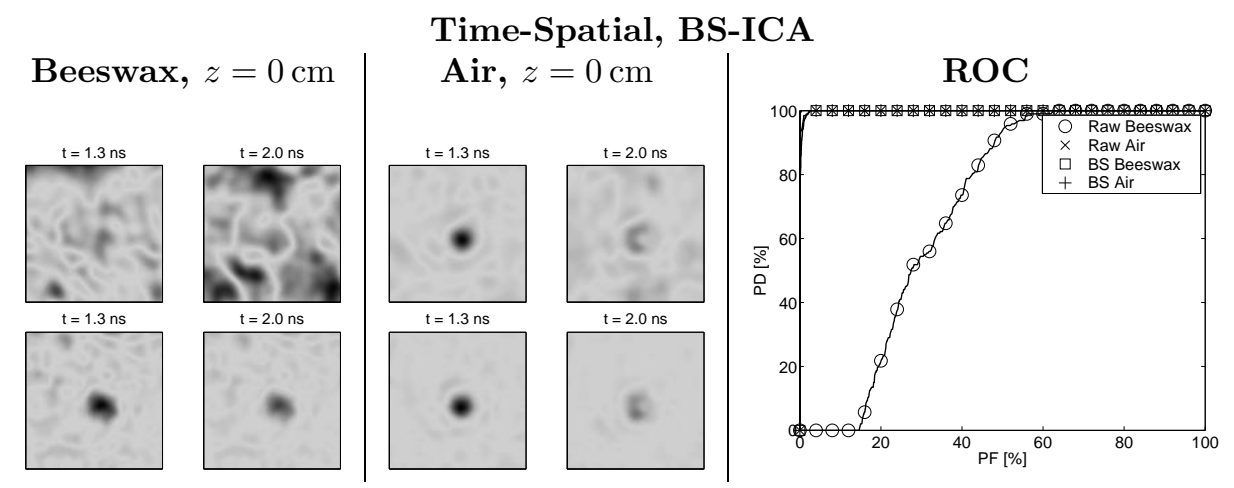

Figure 6. The first row shows the raw SF-GPR data at the time depths $1.3 \mathrm{~ns}$ and $2.0 \mathrm{~ns}$ for cyl. no. 1 and no. 2 buried $0 \mathrm{~cm}$. The second row shows the reconstructed data using BS-ICA on the Time-Spatial ICA mixture model. The images and the ROC curves show that the detection is improved.

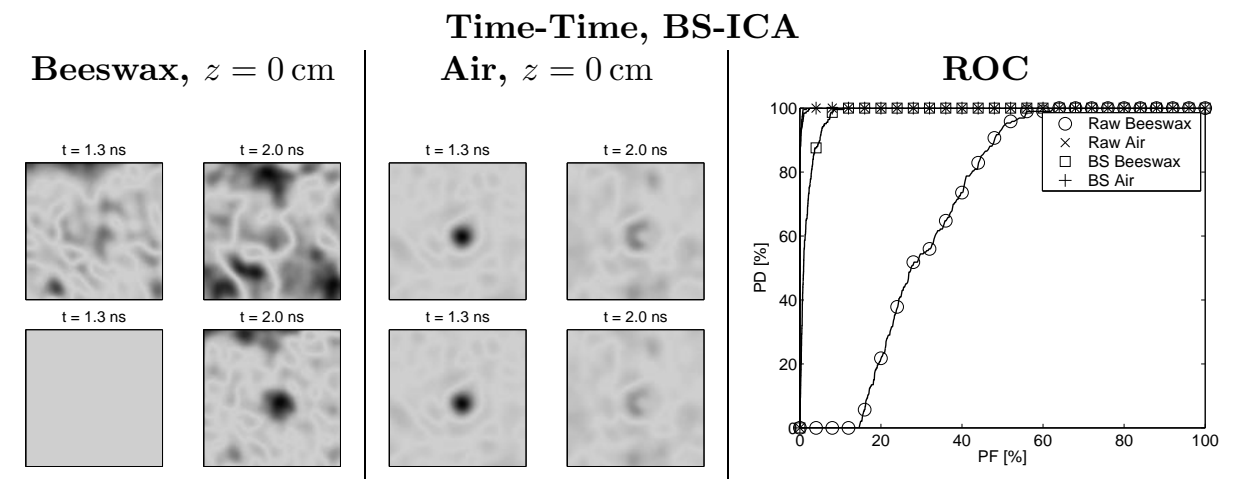

Figure 7. The first row shows the raw SF-GPR data at the time depths $1.3 \mathrm{~ns}$ and $2.0 \mathrm{~ns}$ for cyl. no. 1 and no. 2 buried $0 \mathrm{~cm}$. The second row shows the reconstructed data using BS-ICA on the Time-Time ICA mixture model. The images and the ROC curves show that the detection is improved.

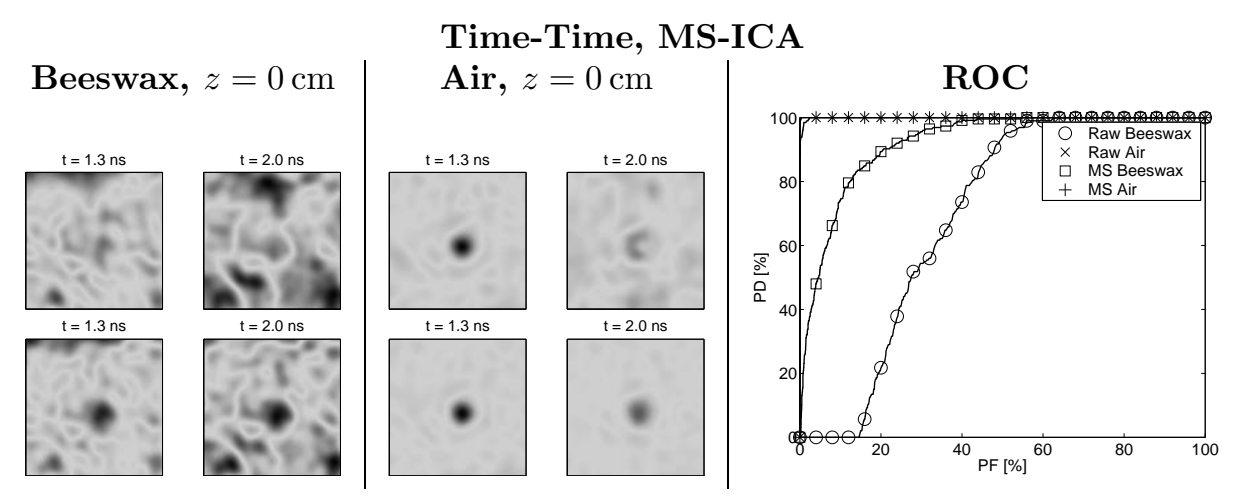

Figure 8. The first row shows the raw SF-GPR data at the time depths $1.3 \mathrm{~ns}$ and $2.0 \mathrm{~ns}$ for cyl. no. 1 and no. 2 buried $0 \mathrm{~cm}$. The second row shows the reconstructed data using MS-ICA on the Time-Time ICA mixture model. The images and the ROC curves show that the detection is improved. 


\begin{tabular}{|c|c|c|c|c|c|c|c|c|c|}
\hline \multirow{2}{*}{ Mode } & \multirow[b]{2}{*}{$\mathbf{z}$} & \multicolumn{4}{|c|}{ Cyl. contents: Beeswax } & \multicolumn{4}{|c|}{ Cyl. contents: Air } \\
\hline & & Raw & PCA & MS-ICA & BS-ICA & Raw & PCA & MS-ICA & BS-ICA \\
\hline \multirow{3}{*}{ F-S } & $0 \mathrm{~cm}$ & 6170 & $9921 / 1$ & $\overline{0.9980 / 1}$ & $9990 / 1$ & .9996 & $9981 / 4$ & $0.9957 / 2$ & $0.9999 / 2$ \\
\hline & $-5 \mathrm{~cm}$ & 7137 & $0.9557 / 2$ & $7499 / 1$ & 999 & 9864 & $9993 / 2$ & $9904 / 2$ & $.9973 / 2$ \\
\hline & $-10 \mathrm{~cm}$ & 0.9363 & $0.8919 / 1$ & $0.9662 / 3$ & $9989 / 1$ & 0.9962 & $\overline{0.9996 / 2}$ & $0.9831 / 2$ & $0.9994 / 2$ \\
\hline \multirow{3}{*}{ F-F } & $\mathrm{cm}$ & 1170 & $\overline{9921 / 1}$ & $9949 / 2$ & & .99 & $\overline{0.9981 / 4}$ & $\overline{0.9999 / 6}$ & $0.9999 / 9$ \\
\hline & $-5 \mathrm{~cm}$ & 0.7137 & $0.9557 / 2$ & 0. & & 0.9962 & $0.9993 / 2$ & $88 / 4$ & $0.9880 / 7$ \\
\hline & $-10 \mathrm{~cm}$ & 0.9363 & $0.8919 / 1$ & $0.9791 / 1$ & & 0.9962 & $0.9996 / 2$ & $0.9997 / 5$ & $0.9648 / 9$ \\
\hline \multirow{3}{*}{ T-S } & $0 \mathrm{~cm}$ & 0.7013 & $0.9843 / 1$ & & $0.9986 / 1$ & 0.9993 & $0.9999 / 1$ & & $0.9999 / 2$ \\
\hline & $-5 \mathrm{~cm}$ & 0.6705 & $0.9104 / 2$ & & $0.9994 / 1$ & 0.9918 & $0.9911 / 3$ & & $35 / 2$ \\
\hline & $-10 \mathrm{~cm}$ & 0.9374 & $0.9643 / 1$ & $0.9498 / 5$ & $0.9985 / 1$ & 0.9751 & $0.9981 / 3$ & & $0.9994 / 2$ \\
\hline \multirow{3}{*}{ T-T } & $0 \mathrm{~cm}$ & 0.7013 & $0.9843 / 1$ & $0.9210 / 3$ & 0.9 & 0.9993 & $0.9999 / 1$ & $0.9999 / 9$ & $0.9994 / 5$ \\
\hline & $-5 \mathrm{~cm}$ & 0.6705 & $0.9104 / 2$ & $0.9355 / 2$ & $0.9506 / 2$ & 0.9918 & $0.9911 / 3$ & $0.9955 / 7$ & $0.9986 / 3$ \\
\hline & $-10 \mathrm{~cm}$ & 0.9374 & $0.9643 / 1$ & & & 0.9751 & $0.9981 / 3$ & $0.9911 / 5$ & $0.9997 / 5$ \\
\hline
\end{tabular}

Table 2. The area under the ROC curve is listed for each SF-GPR example. The closer to 1 the area is the better signal-to-clutter ratio and detection. The number after the stroke for PCA, MS-ICA, and BS-ICA is the number of IC's or PC's containing landmine-like information, and therefore used in reconstruction of the observed signal matrix. The numbers in bold are the best results for each model. Notice for the Frequency-Frequency ICA mixture rather few IC' are extracted. Also notice that the deeper the cylinder is buried the better detection. This is particular shown in the raw data, due to the fact that deeper buried objects are less mixed with the ground surface. Hence, the ground surface is easier to reduce by mean value subtraction.

\section{CONCLUSION}

This paper provides a comparative study of frequency-domain and time-domain based ICA for clutter reduction and non-metallic landmine detection of SF-GPR signals. Four different ICA mixture models were considered: Time-Time (time independence of time signals), Time-Spatial (spatial independence of time signals), FrequencyFrequency (frequency independence of frequency domain signals), and Frequency-Spatial (spatial independence of frequency domain signals). Two state-of-the-art of ICA algorithms Infomax (Bell-Sejnowski) and delayed decorrelation (Molgedey-Schuster) were deployed. The experimental results indicate very different extracted independent components. Moreover, some of the ICA mixture models are not working in a satisfactory way and produce non-physical components. This is particularly the case of using BS-ICA on the Frequency-Frequency ICA mixture model and of using MS-ICA on the Time-Spatial ICA mixture model. The sources are ranked using kurtosis based scheme described in section 4. The ranking shows that landmine information is contained in the first components, and can be used to improve the selection of relevant independent components. The methods were tested on relatively large anti-personnel landmine dummies. Experiments with more realistically sized anti-personnel landmine dummies (diameter $5 \mathrm{~cm}$ )) was unsuccessful since the scattering from the object was too weak using the current GPR acquisition system.

\section{ACKNOWLEDGEMENT}

We thank Ole Nymann for enthusiastic and steady support of our work in humanitarian landmine detection. Furthermore, Peter Meincke and Hans-Rudolf Lenler-Eriksen are acknowledged for collaboration on field data acquisition and the indoor GPR measurement facility at the Technical University of Denmark.

\section{REFERENCES}

1. J.T. Dean: Minetest - Test Support to RESD Actions, International Projects and Related Technical Workshops, Technical Report, Joint Research Center, 2002.

2. D.J. Daniels: Surface Penetrating Radar, IEE, 1996.

3. H.B.D. Sørensen, K.B. Jakobsen and O. Nymann: "Identification of mine-shaped objects based on an efficient phase stepped-frequency radar approach" in Proc. IEEE Image Processing 1997, 3, pp. 142-145. 
4. H. Brunzell: "Clutter Reduction and Object Detection in Surface Penetrating Radar," in Proc. of IEE Radar'97, issue 449, 1997, pp. 688-691.

5. J.W. Brooks, L. van Kempen \& H. Sahli: "Primary Study in Adaptive Clutter Reduction and Buried Minelike Target Enhancement from GPR Data," in Proc. of SPIE, AeroSense 2000: Det. and Rem. Techn. for Mines and Minelike Targets V, vol. 4038, 2000, pp. 1183-1192.

6. L. van Kempen, H. Sahli, E. Nyssen \& J. Cornelis: "Signal Processing and Pattern Recognition Methods for Radar AP Mine Detection and Indentification," Det. of Aband. Land Mines, no. 458, pp. 81-85, 1998.

7. A. van der Merwe \& I.J. Gupta: "A Novel Signal Processing Technique for Clutter Reduction in GPR Measurements of Small, Shallow Land Mines," IEEE Transactions on Geoscience and Remote Sensing vol. 38, no. 6, pp. 2627-2637, 2000.

8. J.L. Salvati, C.C. Chen \& J.T. Johnson: "Theoretical Study of a Surface Clutter Reduction Algorithm," in Proc. of 1998 IEEE International Geoscience and Remote Sensing, vol. 3, 1998, pp. 1460-1462.

9. D. Carevic: "Clutter Reduction and Target Detection in Ground Penetrating Radar Data Using Wavelets," in Proc. of SPIE Conf. on Det. and Rem. Tec. for Mines and Minel. Targ. IV, vol. 3710, 1999, pp. $973-997$.

10. H. Deng \& H. Ling: "Clutter Reduction for Synthetic Aperture Radar Images Using Adaptive Wavelet Packet Transform," in Proc. of IEEE Int. Ant. and Propaga. Soc. Symp., vol. 3, 1999, pp. 1780-1783.

11. A.H. Gynatilaka \& B.A. Baertlein: "A subspace decomposition technique to improve GPR imaging of antipersonnel mines," in Proc. of SPIE, AeroSense 2000: Detect. and Rem. Techn. for Mines and Minelike Targets $V$, vol. 4038, 2000, pp. 1008-1018.

12. B. Karlsen, J. Larsen, K.B. Jakobsen, H.B.D. Sørensen \& S. Abrahamson: "Antenna Characteristics and AirGround Interface Deembedding Methods for Stepped-Frequency Ground Penetrating Radar Measurements," in Proc. of SPIE, AeroSense 2000: Detect. and Rem. Techn. for Mines and Minelike Targets V, vol. 4038, 2000, pp. 1420-1430.

13. B. Karlsen, J. Larsen, H.B.D. Sørensen and K.B. Jakobsen: "Comparison of PCA and ICA based Clutter Reduction in GPR Systems for Anti-Personal Landmine Detection," in Proc. of 11th IEEE Workshop on Statistical Signal Processing, Singapore, Aug. 6-8, 2001, pp. 146-149.

14. A.K. Shaw \& V. Bhatnagar: "Automatic Target Recognition Using Eigen-Templates," in Proc. of SPIE Conference on Algorithms for Synthetic Aperture Radar Imagery V, vol. 3370, 1998, pp. 448-459.

15. S.H. Yu \& T.R. Witten: "Automatic Mine Detection based on Ground Penetratinig Radar," in Proc. of SPIE Conf. on Det. and Rem. Techn. for Mines and Minelike Targets IV, vol. 3710, 1999, pp. 961-972.

16. T.W. Lee: Independent Component Analysis: Theory and Applications Kluwer Academic Publishers, ISBN $0792382617,1998$.

17. A. Cichocki and S.-i. Amari: Adaptive Blind Signal and Image Processing: Learning Algorithms and Applications, John Wiley, Chichester, UK, April, 2002.

18. A. Bell \& T.J. Sejnowski: "An Information-Maximation Approach to Blind Separation and Blind Deconvelution," Neural Computation, vol. 7, pp. 1129-1159, 1995.

19. L. Molgedey \& H. Schuster: "Separation of Independent Signals using Time-Delayed Correlations," Physical Review Letters, vol. 72, no. 23, pp. 3634-3637, 1994.

20. L.K. Hansen, J. Larsen \& T. Kolenda: "On Independent Component Analysis for Multimedia Signals," in L. Guan, S.Y. Kung \& J. Larsen (eds.) Mult. Image and Vid. Proc., CRC Press, Ch. 7, pp. 175-199, 2000.

21. T. Kolenda, S. Sigurdsson, O. Winther, L.K. Hansen \& J. Larsen: DTU:Toolbox, ISP group, Informatics and Mathematical Modelling, Technical University of Denmark, http://isp.imm.dtu.dk/toolbox/, 2002.

22. B. Lautrup, L.K. Hansen, I. Law, N. Mørch, C. Svarer \& S.C. Strother: "Massive weight sharing: A Cure for Extremely Ill-posed Problems," in H.J. Herman et al., (eds.) Supercomputing in Brain Research: From Tomography to Neural Networks, World Scientific Pub. Corp. pp. 137-148, 1995.

23. S. Vorobyov \& A. Cichocki: "Blind Noise Reduction for Multisensory Signals using ICA and Subspace Filtering, with Application to EEG Analysis," in Biological Cybernetics, vol. 86, pp. 293-303, 2002.

24. H.R. Lenler-Eriksen, P. Meincke \& E. Jørgensen: "Estiamtion of Constitutive Parameters Using a Loop Antenna," in Proc. of 2nd International Workshop on Advanced Ground Penetrating Radar (IWAGPR), Delft University of Technology, May 2003. 